\title{
Preparação de arquivos climáticos futuros para avaliação dos impactos das mudanças climáticas no desempenho termoenergético de edificações
}

\author{
Preparation of future weather files to evaluate the \\ impact of climate change on the thermoenergetic \\ performance of buildings
}

\section{Bruna Gomes Casagrande Cristina Engel de Alvarez}

\begin{tabular}{|c|c|}
\hline & Resumo \\
\hline & $\begin{array}{l}\text { rande parte dos estudiosos do clima reconhece que o comportamento } \\
\text { dele é variável e, de acordo com modelos climáticos, uma alteração na } \\
\text { distribuição do calor vem acontecendo desde o século XX. A } \\
\text { mitigação das consequências das mudanças climáticas é pauta de }\end{array}$ \\
\hline & $\begin{array}{l}\text { Palavras-chave: Mudanças climáticas. Eficiência energética. Arquivo climático futuro. } \\
\text { Simulações. }\end{array}$ \\
\hline & Abstract \\
\hline & $\begin{array}{l}\text { Most climatologists recognize that climate behaviour is variable and, according to } \\
\text { climate models, a change in heat distribution has been taking place since the } \\
\text { twentieth century. The mitigation of the effects of climate change is in the public } \\
\text { policy agenda of many countries, and research to find ways of adapting to new } \\
\text { scenarios is being developed in several fields of knowledge. It is known that the } \\
\text { thermal and energy performance of buildings, which are designed for a lifespan of } \\
\text { approximately fifty years, is directly related to the climate to which they are }\end{array}$ \\
\hline $\begin{array}{r}\text { Bruna Gomes Casagrande } \\
\text { Universidade Federal do Espírito } \\
\text { Santo }\end{array}$ & $\begin{array}{l}\text { exposed. Therefore, the consideration of climate change is essential in the } \\
\text { evaluation of the performance of buildings with regards to their energy }\end{array}$ \\
\hline Vitória - ES - Brasil & $\begin{array}{l}\text { consumption. This research study aims to investigate the future performance of } \\
\text { buildings, through the adoption of a method for preparation of weather files }\end{array}$ \\
\hline Cristina Engel de Alvarez & representing future conditions for the city of Vitoria, located in the state of Espirito \\
\hline $\begin{array}{r}\text { Universidade Federal do Espírito } \\
\text { Santo }\end{array}$ & $\begin{array}{l}\text { Santo, Brazil. The suitability of the data was tested through performance analysis } \\
\text { of a commercial building, simulated with the DesignBuilder software. The }\end{array}$ \\
\hline Vitória - ES - Brasil & $\begin{array}{l}\text { capacity of the files to represent future situations was made evident after } \\
\text { examination of the output data, which showed an increase in average annual }\end{array}$ \\
\hline Recebido em 15/04/13 & energy consumption of $10.7 \%$ for 2020, $17.3 \%$ for 2050 and $26.5 \%$ for 2080. \\
\hline Aceito em 04/09/13 & Keywords: Climate change. Energy efficient. Future weather file. Simulation. \\
\hline
\end{tabular}

CASAGRANDE, B. G.; ALVAREZ, C. E. de. Preparação de arquivos climáticos futuros para avaliação dos impactos das mudanças climáticas no desempenho termoenergético de edificações. Ambiente Construído, Porto Alegre, v. 13, n. 4, p. 173-187, jul./set. 2013.

ISSN 1678-8621 Associação Nacional de Tecnologia do Ambiente Construído. 


\section{Introdução}

Considera-se como um dos principais desafios a serem enfrentados pela sociedade do século XXI enfrentar as mudanças no clima em âmbito mundial, especialmente em função das consequências que devem causar ao meio ambiente, à saúde humana e à economia. Na busca pela compreensão do funcionamento do sistema climático, bem como das possíveis mudanças a ocorrer no curto e longo prazos, a climatologia busca desenvolver modelos matemáticos que representem todos os componentes e interações atuantes nesse complexo conjunto de variáveis. Nesse sentido, entende-se o termo "modelos climáticos" como as representações matemáticas do sistema climático que representem seus

\section{[...] principais processos físicos $e$ dinâmicos, assim como as interações entre as componentes do sistema climático e os mecanismos de retroalimentação (feedbacks) entre os processos físicos [...] (MARENGO, 2007, p. 20).}

Com o intuito de fornecer informações amparadas por visões científicas a respeito do clima e desses modelos climáticos, foi criado, em 1988, o Painel Intergovernamental sobre Mudanças Climáticas (IPCC) pela Organização Meteorológica Mundial e pelo Programa das Nações Unidas para o Meio Ambiente. Em síntese, esse órgão tem como principal objetivo estudar as bases científicas, técnicas e socioeconômicas relevantes para a compreensão dos riscos das mudanças climáticas induzidas ao homem, seus potenciais impactos e opções para adaptação e mitigação (INTERGOVERNMENTAL..., 2010).

Desde então, o IPCC tem publicado relatórios científicos que condensam informações sobre as alterações climáticas coletadas pelas distintas instituições colaboradoras de diversas regiões do mundo (MARENGO, 2007). Nesses relatórios são apresentados, também, os dados obtidos através da construção dos MCGs (Modelos de Circulação Geral), ressaltando-se que este é o tipo mais complexo de modelo climático, pois consideram a maioria dos processos físicos relevantes para o clima e abordam-nos em três dimensões: a latitude, a longitude e a altura. Além disso, esses modelos geralmente acoplam um MCG oceânica, dada a importância do comportamento do oceano sobre o clima (PITA, 2006)

As características demográficas, socioeconômicas e tecnológicas futuras, bem como as variações nas emissões de substâncias com efeito radiativo potencial a elas associadas, são representadas nos MCGs através dos chamados "cenários de emissão" (MARENGO, 2007). A configuração desses cenários procura a representação de situações futuras a partir de uma perspectiva mais otimista (denominadas famílias B1 e B2), com ênfase para soluções que visem à sustentabilidade econômica, social e ambiental, e de projeções mais pessimistas (denominadas famílias A1 e A2). O cenário A1 projeta um futuro globalizado, caracterizado pelo rápido crescimento econômico, pequeno crescimento populacional e desenvolvimento rápido de tecnologias mais eficientes. O cenário A2 é caracterizado pelo regionalismo socioeconômico e cultural, além do alto crescimento populacional e do lento desenvolvimento tecnológico.

Apesar de alguns climatologistas discordarem do conteúdo publicado nesses relatórios, principalmente a respeito dos fenômenos apontados como determinantes para as alterações do clima, grande parte da comunidade científica corrobora as proposições sobre o comportamento variável do sistema climático (AMBRIZZI; ARTAXO, 2012). De acordo com as informações geradas por modelos climáticos globais e regionais, uma anormalidade na distribuição do calor entre as regiões do planeta vem acontecendo desde o século XX e, por conseguinte, pesquisas para adequação aos novos cenários têm sido desenvolvidas em diversas áreas do conhecimento.

Sabe-se que a edificação oferece uma interface entre o ambiente externo - sujeito às mudanças climáticas - e o ambiente interno, que precisa ser mantido em condições tais que ofereçam conforto e segurança aos usuários. Atualmente, a parcela de energia consumida para manter as edificações confortáveis é considerada uma oportunidade para diminuição do desperdício do total produzido, uma vez que a adoção de estratégias construtivas adequadas pode reduzir o consumo final de eletricidade. Adquire fundamental importância, portanto, a investigação do desempenho de edificações quanto às demandas energéticas futuras, particularmente durante o ciclo de vida planejado para cada edifício.

Graças ao avanço da tecnologia computacional, o comportamento térmico e energético de um edifício pode ser avaliado através de ferramentas virtuais, que permitem a inserção de uma grande quantidade de variáveis, representantes das características reais da edificação que se pretende investigar. O Departamento de Energia dos Estados Unidos, por exemplo, disponibiliza informações sobre 403 softwares para simulação e avaliação de eficiência energética, energias renováveis e sustentabilidade em edificações 
(UNITED..., 2013). Para a transformação dos dados climáticos do local onde estiver inserida a edificação em um formato compatível com tais ferramentas de simulação, existem vários procedimentos ou metodologias passíveis de serem aplicadas, sendo a tarefa de preparação dos arquivos - com a confiabilidade esperada para os procedimentos posteriores - um dos desafios da pesquisa.

Diante do exposto, esta pesquisa teve por objetivo investigar o desempenho futuro de edificações, através da adoção de um método para preparação de arquivos climáticos, considerando serem dados fundamentais para as simulações termoenergéticas de edificações, referentes ao clima futuro, sendo o recorte territorial a cidade de Vitória, ES. O teste da adequabilidade dos dados foi efetuado mediante a análise do comportamento de um edifício comercial em três períodos futuros, através de simulações de oito modelos no software DesignBuilder.

\section{Estudos sobre mudanças climáticas no Brasil}

De acordo com Marengo (2007), com o intuito de entender a variabilidade do clima atual do Brasil e suas possíveis mudanças futuras, foi instituído o projeto "Caracterização do clima atual e definição das alterações climáticas para o território brasileiro ao longo do século XXI", pelo Centro de Previsão de Tempo e Estudos Climáticos do Instituto Nacional de Pesquisas Espaciais e colaboradores. Essa pode ser considerada uma das primeiras investigações sobre o comportamento do clima futuro no Brasil e tem como objetivo estimular o desenvolvimento de pesquisas sobre o tema, inclusive daquelas relacionadas aos impactos e à vulnerabilidade dos diferentes setores sociais e ambientais (CENTRO..., 2005).

Com relação ao clima do futuro, os resultados dessa pesquisa indicaram que a temperatura média no ar à superfície, dependendo do cenário de emissão de gases de efeito estufa e dos modelos climáticos globais, pode sofrer um aumento de 4 ${ }^{\circ} \mathrm{C}$ acima da média climatológica (1961-1990) para 2100, com consideráveis variações regionais. Embora ainda existam incertezas nessas projeções - mais ou menos acentuadas de acordo com o parâmetro climático ou a região -, diversas hipóteses são levantadas com relação aos regimes e distribuição de chuva, ocorrência de eventos extremos, amplitude do fenômeno El Niño, variações no ciclo hidrológico, entre outros (MARENGO, 2007).
Essas projeções implicam uma série de consequências à sociedade, lembrando que aqueles que hoje dispõem de menos recursos terão menor capacidade de adaptação. Nesse sentido, o Nordeste é considerado a região mais vulnerável do Brasil, pois a atual estação chuvosa fundamental para a agricultura de subsistência regional, para a disponibilidade de água e para a saúde da população - provavelmente não existirá num clima mais quente.

Relacionam-se, ainda, outras questões preocupantes nesses relatórios brasileiros, tais como: vulnerabilidade de crianças e idosos às possíveis ondas de calor; morte de recifes de corais; agravamento do problema das ilhas de calor urbanas; perda de biodiversidade e de recursos naturais; aumento do risco de incidência de doenças como malária, dengue, febre amarela e encefalite, além de doenças respiratórias; entre outros (CENTRO..., 2005).

Nesse sentido, no meio acadêmico diversas áreas do conhecimento têm considerado efetivamente as mudanças projetadas para o clima em suas pesquisas, seja para a identificação dos possíveis impactos, seja para a proposição das ações para mitigação dessas consequências (FREITAS; AMBRIZZI, 2012). É importante ressaltar a importância dos estudos climatológicos nesse contexto, uma vez que fornecem as bases - dados climatológicos - para o desenvolvimento de situações futuras de grande parte dos estudos mencionados anteriormente.

Experimentos científicos também são realizados para uma melhor compreensão dos ecossistemas na América do Sul. A Amazônia concentra um grande número de pesquisas, tanto para o entendimento de sua influência sobre o clima global, como para avaliação das ações antrópicas sobre aquele território. Pesquisadores brasileiros também investigam a criosfera por meio do Instituto Nacional de Ciência e Tecnologia da Criosfera e seu papel para a formação climática atual e futura (INSTITUTO..., 2013).

Além dos estudos climatológicos que objetivam, geralmente, identificar anomalias nas sequências de dados que indiquem uma possível alteração nos padrões do clima, estudos ligados às ciências da terra destacam-se nessa temática. Devido à maior suscetibilidade a mudanças climáticas, estudos da área agrícola procuram antecipar as consequências sobre as mais diversas culturas, aliando-se, inclusive, os impactos à economia (FREITAS; AMBRIZZI, 2012).

Outra área cuja importância é ressaltada em estudos sobre a adaptação da sociedade às mudanças climáticas é a saúde. Algumas pesquisas 
relatam que as grandes metrópoles são regiões de maior vulnerabilidade, em virtude de suas características sociodemográficas e geográficas. Acidentes e traumas também devem ser considerados, devido à ocorrência de eventos extremos, como furacões, inundações, deslizamentos de encostas, entre outros (CONFALONIERI, 2012).

As alterações no regime de chuvas, e consequentemente nas vazões dos rios, estão na pauta não somente de estudos acerca de eventos extremos, mas também das pesquisas sobre a oferta de energia elétrica nacional (BARROS, 2012). O quadro energético brasileiro atual apresenta oito diferentes fontes para produção de energia elétrica, sendo que $74 \%$ do total produzido correspondem à energia gerada por hidrelétricas (MINISTÉRIO..., 2011), que dependem, em última instância, da água das chuvas. Por isso, apesar de a hidroeletricidade representar um sistema de produção de energia com fator mínimo de emissão de dióxido de carbono (BARROS, 2012), as mudanças no comportamento climático futuro, principalmente suas consequências para o sistema hídrico, podem impactar indiretamente o fornecimento de energia elétrica em âmbito nacional.

Diante do cenário apresentado, é perceptível que os programas para eficientização do consumo de energia devam assumir um papel fundamental no desenvolvimento de políticas para a adaptação da sociedade às mudanças climáticas futuras.

\section{Estudos sobre o impacto das mudanças climáticas em edifícios}

Pesquisas sobre o impacto das mudanças climáticas sobre edifícios têm um histórico relativamente recente, considerando-se que foram intensificadas aproximadamente a partir de 2000 (DE WILDE; TIAN, 2011). Anteriormente, o objetivo de estudos relacionando mudanças climáticas e edifícios concentrava-se, fundamentalmente, no agravamento das condições climáticas futuras ocasionado pelo aumento no consumo de energia por edificações, por corresponder a um aumento nas emissões de gases de efeito estufa. A "resposta" dos edifícios a essas alterações passou posteriormente à condição de objeto de grande parte das pesquisas dessa área.

Atualmente, uma das questões extensivamente exploradas em pesquisas sobre impacto de mudanças climáticas em edifícios é o método de transformação de arquivos climáticos, considerando ser um dado de entrada fundamental para softwares de simulação. Ressalta-se que o aprimoramento de métodos de avaliação do desempenho termoenergético de edificações atingiu seu ápice com a maior facilidade de uso desses softwares, visto que eles, em sua maioria, permitem uma descrição detalhada do objeto de estudo, além da inserção de maior quantidade de informações sobre o clima com o qual se relaciona. Assim, a consideração de eventos futuros na preparação de arquivos climáticos permite uma representação mais próxima ao cenário com o qual o edifício irá interagir, destacando-se que usualmente tais arquivos são formatados de acordo com as características do clima atual ou de períodos passados.

Embora a inserção de informações climáticas projetadas para o futuro em arquivos climáticos seja uma técnica relativamente recente, um número considerável de pesquisadores tem aplicado esse método para estudar o desempenho futuro dos espaços construídos. Nesse panorama, o método morphing destaca-se entre diversas publicações, tais como Jentsch, Bahaj e James (2008), Guan (2009), De Wilde e Tian (2009), Wang, Chen e Ren (2010), Chan (2011b), Kolokotroni et al. (2012) e Robert e Kummert (2012).

A metodologia morphing, publicada pela Chartered Institution of Building Services Engineers (CIBSE), foi desenvolvida originalmente para transformar arquivos climáticos TRY (Test Reference Year) atuais em arquivos climáticos futuros, considerando-se as mudanças projetadas por MCGs desenvolvidos no Reino Unido. Os dados climáticos horários presentes no arquivo TRY são ajustados para representar uma possível condição futura através dos valores mensais de algumas variáveis climáticas, projetados por um modelo climático para a respectiva região (BELCHER; HACKER; POWELL, 2005).

Adaptações da metodologia morphing foram apresentadas posteriormente, geralmente com fornecimento de indicativos voltados para a disponibilidade de dados de cada localidade. No caso da Austrália, por exemplo, algumas variáveis climáticas possuem projeções futuras mais detalhadas que outras, tendo sido desenvolvido um processo de modificação para cada variável, dependendo do nível de informações disponíveis sobre ela (GUAN, 2009).

Além disso, conhecendo as incertezas inerentes aos processos de modelagem climática, bem como a variação entre as configurações dos modelos, algumas pesquisas procuram a aplicação de técnicas que atenuem tais dubiedades. Uma dessas técnicas é a consideração das projeções futuras de

176 Casagrande, B. G.; Alvarez, C. E. de. 
mais de um modelo climático, para posterior associação aos dados atuais (WANG; CHEN; REN, 2011). Destaca-se, no entanto, que a manipulação desses dados exige um conhecimento específico e aprofundado em ciências climatológicas, o que pode ser um obstáculo para o desenvolvimento dessas pesquisas, visto que os arquivos climáticos existentes são, geralmente, formatados pelos próprios pesquisadores do ambiente construído. Além disso, MCGs demandam grande quantidade de tempo e equipamentos de alta capacidade de processamento para a obtenção de resultados. No entanto, já é perceptível o interesse pelo assunto por vários profissionais de diversificadas áreas do conhecimento, sugerindo que, em breve, a necessidade de conhecimento específico será ultrapassada.

No Reino Unido, a diversidade de pesquisas relacionadas ao impacto das mudanças climáticas sobre edifícios, oportunizada principalmente pelos avançados estudos climatológicos da região, possibilitou o aprimoramento dos métodos de preparação de arquivos climáticos futuros. Com uma ferramenta desenvolvida pelo Climate Impacts Programme, arquivos climáticos são formatados com dados probabilísticos das variáveis futuras, diminuindo o grau de incertezas em comparação à utilização de projeções de MCGs (EAMES; KERSHAW; COLEY, 2012).

Ainda sobre o preparo de arquivos climáticos futuros, uma abordagem complementar do assunto, originada da preocupação com a adaptação futura dos edifícios ao clima, é a probabilidade de ocorrência de eventos extremos, assunto bastante discutido devido à urgência para investigação das formas de mitigação de suas consequências. Em estudos sobre edificações é usual o ajuste das informações climáticas a partir de arquivos com formato TRY, impondo a desconsideração de eventos extremos passados e deixando de projetálos no futuro, uma vez que seu processo metodológico constitui-se justamente na eliminação de anos com temperaturas médias mensais extremas. Por esse motivo algumas pesquisas têm atuado não somente na inserção de informações climáticas futuras, como também na reformulação de dados de períodos anteriores, passando a considerar um maior intervalo de tempo como base para as transformações, inclusive seus eventos extremos (DU; EDGE; UNDERWOOD, 2011; KERSHAW; EAMES; COLEY, 2011; ROBERT; KUMMERT, 2012).

Essa preocupação com a representação minuciosa e precisa do meio em que o edifício se insere em softwares de simulação termoenergética tem motivado não só o ajuste de informações climáticas futuras como também a inserção de dados climáticos que registrem as ilhas de calor urbanas, fenômeno observado atualmente na maioria das grandes cidades (KOLOKOTRONI et al., 2012). Para comparar o desempenho de edifícios localizados em áreas urbanas e rurais, Chan (2011a) aplicou a metodologia morphing, associando dados medidos em pontos do centro de Hong Kong ao arquivo climático histórico. Já Crawley (2007) buscou registros bibliográficos para representar o efeito das ilhas de calor das 20 regiões consideradas em sua pesquisa. Além das alterações projetadas por um MCG, o autor considera que as ilhas de calor provocam variações de $1^{\circ} \mathrm{C}$ a $5^{\circ} \mathrm{C}$ na temperatura do ar.

Constata-se, portanto, que um considerável número de pesquisas tem-se concentrado na preparação de arquivos climáticos futuros com a finalidade de, sobretudo, investigar estratégias projetuais adequadas não só às condições climáticas atuais mas, principalmente, aos cenários futuros.

Algumas pesquisas procuram, unicamente, comprovar a necessidade de consideração das condições climáticas futuras nos projetos mediante estudos de caso, enfatizando que toda e qualquer construção deve ser planejada para o futuro, e não para o passado (LEE, 2011; ROBERT; KUMMERT, 2012). De acordo com cada localidade, diversas hipóteses são levantadas sobre o desempenho futuro dos espaços construídos atualmente, na tentativa de identificar quais dessas estratégias serão válidas para o bom funcionamento do edifício durante sua vida útil.

Com base em levantamentos do setor energético do Reino Unido, por exemplo, verificou-se um grande potencial para redução do consumo de energia do setor residencial, motivando a investigação da eficiência de sistemas construtivos considerados, atualmente, adequados às condições climáticas locais. Com esse estudo confirmou-se a importância da utilização de vidros duplos nas aberturas, pois contribuem significativamente para a economia do consumo de energia para aquecimento, tanto para condições climáticas atuais quanto futuras (GATERELL; MCEVOY, 2005). Na Austrália, edifícios residenciais considerados eficientes por um sistema de avaliação apresentaram-se mais sensíveis às mudanças climáticas que edifícios menos eficientes, tendo o incremento no consumo de energia projetado para 2050 variado entre $26 \%$ e $101 \%$, e para 2100 , entre $48 \%$ e $350 \%$ (WANG; CHEN; REN, 2010).

Em Hong Kong, estudos apontam um aumento substancial no consumo de energia para 
condicionamento artificial do ar, variando de $2,6 \%$ a $14,3 \%$ para edifícios de escritório e de $3,7 \%$ a $24 \%$ para residências (CHAN, 2011b). Em Burkina Faso, país localizado na região noroeste na África, o aumento no consumo de energia para resfriamento previsto é de $56 \%$ entre 2030 e 2049 , e de $99 \%$ entre 2060 e 2079 , em comparação ao período entre 2010 e 2029 (OUEDRAOGO; LEVERMORE; PARKINSON, 2012). A pesquisa conclui que a orientação do edifício, a área total envidraçada, a transmitância de elementos opacos e dispositivos de sombreamento são algumas estratégias que contribuem significativamente para a definição desses valores. Edifícios com a classificação net-zero - ou seja, aqueles que produzem a própria energia que irão consumir também passaram a ser discutidos quanto a seu desempenho futuro, e no Canadá um estudo de caso aponta que o edifício não conseguirá futuramente suprir sua própria demanda de energia (ROBERT; KUMMERT, 2012).

Jentsch, Bahaj e James (2008) concluíram, através do estudo de um edifício de escritórios naturalmente ventilado localizado na cidade de Southampton, no Reino Unido, que um edifício que não utilize condicionamento artificial do ar dificilmente conseguirá manter as condições apropriadas de conforto em seu interior sem a utilização de estratégias construtivas adequadas, como proteção solar externa, uso de massa térmica, estratégias de ventilação bem desenvolvidas, entre outros. Um aumento de desconforto pelo calor em edifícios climatizados naturalmente foi observado por Du, Edge e Underwood (2011), ao estudarem o impacto das novas projeções climáticas do Reino Unido sobre o desempenho de um edifício que abriga uma escola primária. Na cidade de Londres, Kolokotroni et al. (2012) constataram que, no verão, o edifício considerado em sua pesquisa já se apresenta desconfortável, quadro que deverá ser intensificado no futuro, exigindo a instalação de resfriamento artificial em edifícios que hoje são climatizados naturalmente. Os autores ressaltam, ainda, a importância dos ganhos internos de calor como um fator significativo para o desempenho energético futuro. Hanby e Smith (2012) demonstram, através de estudos de caso no Reino Unido, que sistemas de resfriamento evaporativo poderão ser uma técnica viável em 2050 para um melhor desempenho termoenergético de edifícios.

\section{Método para configuração de arquivos climáticos futuros}

Os procedimentos metodológicos adotados partiram de uma etapa inicial de reconhecimento do objeto com especial ênfase na revisão bibliográfica dos assuntos inerentes ao tema visando, principalmente, à aquisição do necessário embasamento conceitual para as futuras etapas da pesquisa. Posteriormente, foram identificadas as possíveis ferramentas a serem utilizadas, sendo selecionada a apresentada por Jentsch, Bahaj e James (2008) para conversão de arquivos climáticos atuais em arquivos climáticos futuros. As rotinas internas da ferramenta foram elaboradas, principalmente, a partir do método morphing e são descritas detalhadamente no referencial técnico da ferramenta.

A escolha pela utilização desse método se deu, principalmente, pelos cálculos complementares internos à ferramenta, que executam modificações em todas as variáveis presentes no arquivo climático base, ao contrário de outras metodologias consultadas. Além do método morphing, a ferramenta contempla rotinas desenvolvidas a partir de fontes especializadas em assuntos referentes a cada variável em questão.

Dependendo do parâmetro climático a ser modificado, a metodologia morphing define a aplicação dos seguintes algoritmos (BELCHER; HACKER; POWELL, 2005):

(a) um "desvio" no atual parâmetro climático horário através da adição da variação média mensal projetada, em valores absolutos (Eq. 1):

$x=x_{o}+\Delta x_{m}$

Eq. 1

Onde:

$x$ é a variável climática futura;

$x_{o}$ é a variável original; e

$\Delta x_{m}$ é a anomalia mensal absoluta de acordo com o modelo de projeções utilizado.

Aplica-se, por exemplo, para ajuste da pressão atmosférica;

(b) uma "extensão" no atual parâmetro climático horário através de escalonamento com a variação média mensal projetada, em valores relativos (Eq. 2):

$x=a_{m} x_{o}$

Onde $a_{m}$ é a variação mensal fracionada, de acordo com o modelo de projeções utilizado. Aplica-se, por exemplo, para ajuste da velocidade do vento; e

(c) uma combinação de "desvio" e "extensão" do atual parâmetro climático horário. Neste caso, o parâmetro atual é "desviado" pela adição da anomalia média mensal projetada, em valores absolutos, e "estendido" pela variação diurna mensal do parâmetro (Eq. 3): 
$x=x_{o}+\Delta x_{m}+a_{m}\left(x_{o}-\left(x_{o}\right)_{m}\right)$

Eq. 3

Onde:

$\left(x_{o}\right)_{m}$ é a média mensal relativa à variável $x_{o}$; e $a_{m}$ é a razão entre as variações mensais de $\Delta x_{m} \mathrm{e}$ $x_{o}$. Aplica-se, por exemplo, para ajuste da temperatura de bulbo seco, a fim de integrar as variações do ciclo diurno.

Para conversão dos arquivos climáticos atuais em arquivos futuros, foi utilizada a ferramenta desenvolvida por Jentsch, Bahaj e James (2008), denominada Climate Change World Weather File Generator. A ferramenta é disponibilizada em formato de planilha eletrônica e permite a integração de arquivos de extensão EPW (EnergyPlus/EPSr Weather) ao modelo climático global HadCM3 (Hadley Centre Coupled Model version3). Esse modelo climático foi elaborado pelo Meteorological Office Hadley Centre do Reino Unido para o Terceiro Relatório do IPCC. O HadCM3 é um modelo acoplado oceanoatmosfera, com resolução de $417 \mathrm{~km}$ x $278 \mathrm{~km}$ na região do Equador e de $295 \mathrm{~km}$ x $278 \mathrm{~km}$ aos $45^{\circ}$ de latitude.

O arquivo climático base a ser utilizado nessa ferramenta deve ser, necessariamente, referente ao período entre 1961 e 1990, uma vez que o modelo climático global HadCM3 executa suas projeções a partir desse período. Por esse motivo foi adotado o arquivo climático compilado a partir de dados obtidos da Empresa Brasileira de Infraestrutura Aeroportuária (Infraero) na década de 1990, com formato TRY, para a cidade de Vitória (LABORATÓRIO..., 2005).

Os relatórios brasileiros sobre as mudanças climáticas no Brasil (MARENGO, 2007), embora contribuam para o desenvolvimento de pesquisas em diversas áreas do conhecimento, não apresentam dados suficientes para a configuração de arquivos climáticos com reduzida margem de incertezas. Por esse motivo, optou-se pelo método cuja ferramenta utiliza o modelo global HadCM3.

O cenário de emissões considerado na ferramenta de Jentsch, Bahaj e James (2008) é o A2, e os períodos configurados são selecionados a partir dos anos 2020 (período entre 2011 e 2040), 2050 (período entre 2041 e 2070) e 2080 (período entre 2071 e 2100), e representam mudanças em relação ao período entre 1961 e 1990.

\section{Configuração das variáveis de teste e de controle da edificação}

O objeto a partir do qual foram testados os arquivos climáticos formatados nesta pesquisa é um edifício de uso comercial, hipoteticamente localizado na cidade de Vitória, capital do estado do Espírito Santo (Brasil), que procura agrupar características comuns de edifícios construídos nessa região. A seleção dessa localidade foi motivada por já estarem sendo desenvolvidas pesquisas semelhantes nesse município e por toda a infraestrutura de apoio ao desenvolvimento das atividades - equipamentos e recursos humanos estar vinculada ao Laboratório de Planejamento e Projetos da Universidade Federal do Espírito Santo (LPP/Ufes). O Quadro 1 resume as principais características construtivas desse edifício e as respectivas fontes consultadas para determinação de suas características essenciais.

Quadro 1 - Características construtivas do edifício

\begin{tabular}{|c|c|c|}
\hline \multicolumn{3}{|c|}{ TIPOLOGIA CONSTRUTIVA } \\
\hline Variável de controle & Característica & Fonte \\
\hline Gabarito & 11 pavimentos & Lamberts, Ghisi e Ramos (2006) \\
\hline Pé-direito & $3,00 \mathrm{~m}$ & \multirow{3}{*}{ Bernabé (2012) } \\
\hline Planta & $30 \mathrm{~m} \times 15 \mathrm{~m}$ & \\
\hline Salas & $12 /$ pavimento & \\
\hline Paredes & $\begin{array}{l}\text { Bloco cerâmico, } 6 \text { furos, } \\
15 \mathrm{~cm}\end{array}$ & $\begin{array}{l}\text { Lamberts, Ghisi e Ramos (2006); } \\
\text { Bernabé (2012) }\end{array}$ \\
\hline $\begin{array}{l}\text { Absortância (componentes } \\
\text { opacos das fachadas) }\end{array}$ & 0,4 & \multirow[t]{2}{*}{ Lamberts, Ghisi e Ramos (2006) } \\
\hline Vidros & $6 \mathrm{~mm}$, transparente & \\
\hline Cobertura & Laje protegida por telhado & $\begin{array}{l}\text { Lamberts, Ghisi e Ramos (2006); } \\
\text { ABNT (2005) }\end{array}$ \\
\hline
\end{tabular}


Embasados em uma revisão crítica de publicações recentes sobre o impacto das mudanças climáticas sobre edificações, De Wilde e Tian (2011) enfatizam a necessidade de estabelecimento de limites nessas avaliações. Os autores ressaltam a carência de correlações entre os parâmetros analisados nas pesquisas e a real expectativa de vida das edificações e dos sistemas. É imprescindível a consideração das incertezas nesses processos, pois é altamente improvável que um edifício e seu regime operacional sejam exatamente os mesmos em 50-100 anos. Além disso, a adoção de suposições a respeito da renovação dos componentes da construção requer previsões sobre a evolução tecnológica e a eficiência dos sistemas para 15-20 anos.

Uma das orientações dos autores para pesquisas futuras é, portanto, concentrar-se em aspectos passivos inerentes à construção, como orientação e massa térmica, e que provavelmente não serão afetados por intervenções futuras. Aplicar corretamente esses aspectos reduziria as incertezas sobre as avaliações (DE WILDE; TIAN, 2011).

No Brasil, um dos principais instrumentos para avaliação de questões relacionadas ao desempenho térmico e à eficiência energética de edificações é o Regulamento Técnico da Qualidade do Nível de Eficiência Energética de Edifícios Comerciais, de Serviços e Públicos (RTQ-C). A classificação de um edifício avaliado pelos requisitos presentes nesse regulamento é simbolizada por uma etiqueta, ponderando-se as avaliações de cada sistema em separado: envoltória, iluminação e condicionamento de ar (MINISTÉRIO... 2009a).

Desse modo, a seleção das variáveis a serem investigadas foi fundamentada nos requisitos avaliados pelo RTQ-C (MINISTÉRIO..., 2009b), relacionados à envoltória da edificação e de difícil reestruturação para adequação a novas condições climáticas (Figuras 1 a 6).

\section{Figura 1 - Maiores fachadas orientadas a norte e sul}

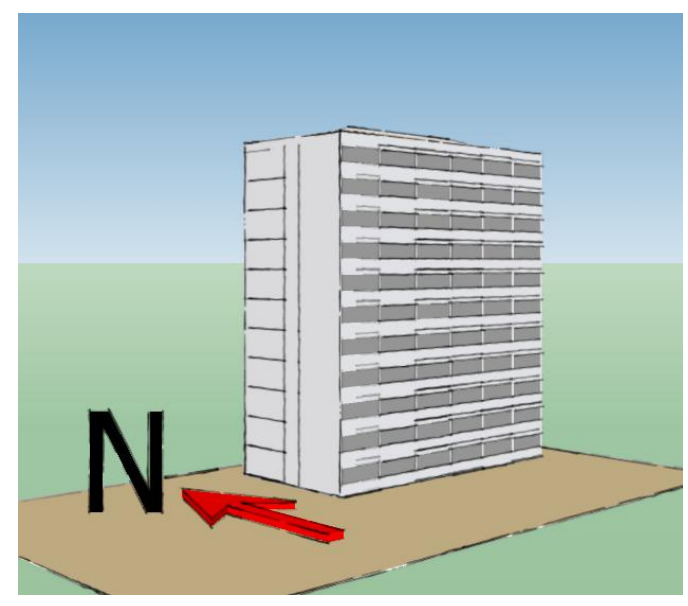

Figura 2 - Maiores fachadas orientadas a leste e oeste

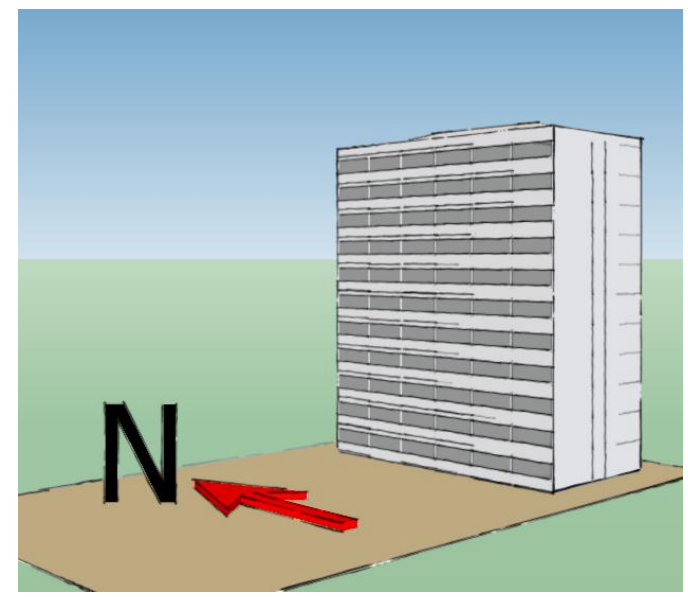

180 Casagrande, B. G.; Alvarez, C. E. de. 
Figura 3 - Edifício com $\mathbf{5 0 \%}$ de abertura

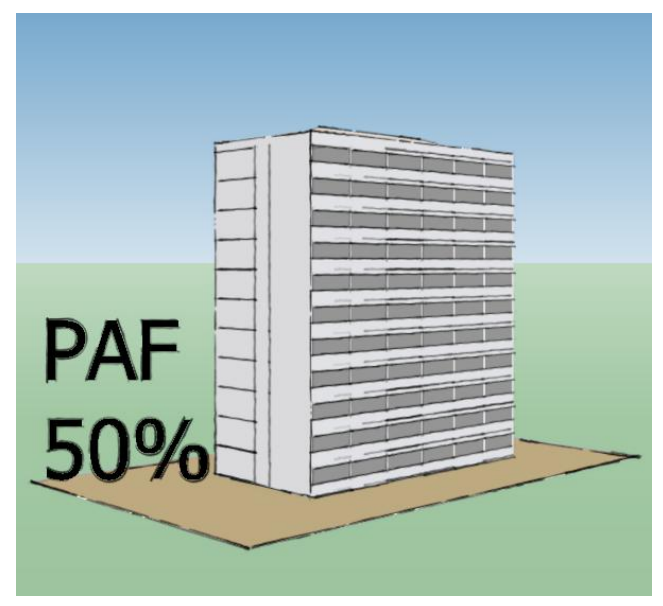

Figura 4 - Edifício com $90 \%$ de abertura

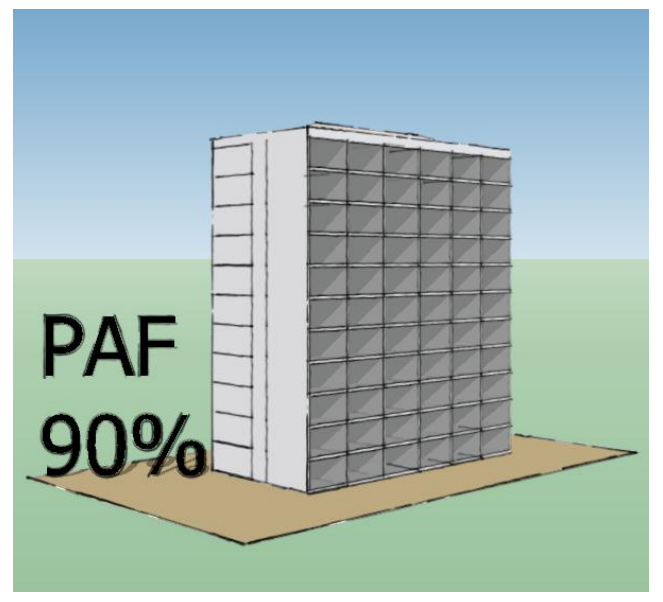

Figura 5 - Edifício sem proteção solar

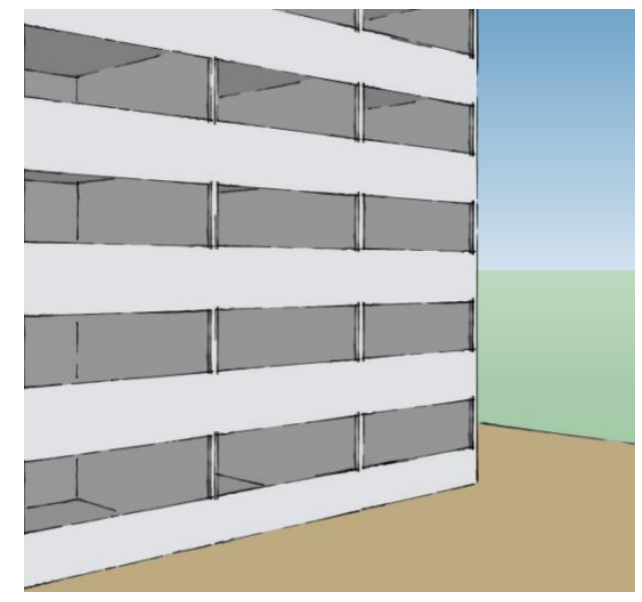


Figura 6 - Edifício com proteção solar

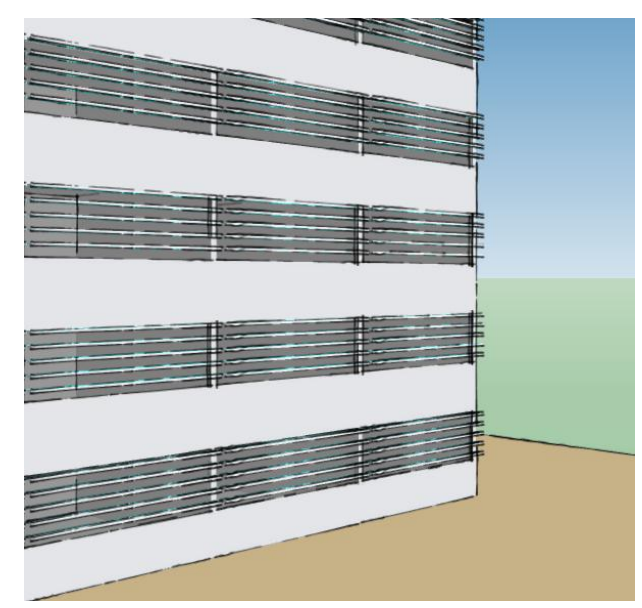

Conforme o MME (MINISTÉRIO..., 2009b), considera-se como percentual de abertura na fachada a "razão entre a soma das áreas de abertura envidraçada, ou com fechamento transparente ou translúcido, de cada fachada e a área total de fachada da edificação". O percentual de abertura adotado nesta pesquisa aplica-se somente às maiores fachadas.

Em resultados publicados em pesquisas anteriores desenvolvidas pela mesma equipe, edifícios com dispositivos de proteção solar específicos para cada orientação e bloqueio da radiação solar direta na maior parte do dia não apresentaram economia no consumo de energia total devido ao aumento no consumo para iluminação (BERNABÉ, 2012). Assim, optou-se pela simplificação dos modelos, utilizando-se de uma mesma configuração de proteção solar, mais simples, para as fachadas protegidas.

Conhecendo a variabilidade de informações necessárias à simulação, a seleção do software foi guiada por critérios que otimizassem o desenvolvimento da pesquisa. O EnergyPlus é uma das ferramentas disponibilizadas pelo Departamento de Energia dos Estados Unidos, e entre suas funções está a análise de aspectos térmicos e energéticos de edificações. A partir da inserção das características do edifício - as quais permitem um detalhamento para que o modelo seja construído o mais próximo possível da realidade -, o software calcula as cargas necessárias para aquecimento e resfriamento para que as temperaturas dos ambientes sejam mantidas dentro de determinado limite (UNITED..., 2013).

Para a realização das simulações foi selecionado, portanto, o software DesignBuilder, considerando ser uma interface gráfica amigável para o EnergyPlus, principalmente pela reprodução tridimensional da geometria da edificação. A combinação de todas as variáveis de teste originou oito diferentes modelos a serem simulados em quatro períodos - atual, 2020, 2050 e 2080 resultando em 32 simulações.

\section{Resultados e discussão}

Após o processamento das simulações, procedeuse à análise dos consumos totais anuais - com climatização, iluminação e equipamentos - e sua evolução ao longo do século XXI. Considerandose o conjunto dos oito modelos, observou-se um aumento médio no consumo anual de $10,7 \%$ para o período de $2020,17,3 \%$ para o período de 2050 , e $26,5 \%$ para o período de 2080 , em relação ao consumo atual.

A tendência de aumento do consumo pode ser observada na Figura 7, em comparação ao aumento das temperaturas de bulbo seco. As médias mensais de aumento no consumo de energia apresentam comportamento semelhante ao aumento das temperaturas. Observa-se, ainda, um aumento no consumo mais acentuado que o aumento das temperaturas nos meses de verão.

O edifício orientado a leste e oeste, com $90 \%$ de abertura e sem proteção solar (Figura 8), apresenta o maior consumo atual e nos períodos futuros. $\mathrm{O}$ edifício com menor consumo atual também permanece com o menor consumo nas etapas futuras e está orientado a norte e sul, possui $50 \%$ de abertura e dispositivos de proteção solar.

A alteração da orientação causou maior diferença entre as taxas médias de aumento do consumo que a alteração do percentual de abertura ou a utilização de dispositivos de proteção solar (Figura 9). Os edifícios orientados a norte e sul mostraram, em média, menores taxas de aumento que os edifícios orientados a leste e oeste. A variação do percentual de abertura na fachada não provocou 
alterações tão acentuadas, comparativamente, nas taxas de aumento do consumo, sendo que em 2080 essas taxas quase se equiparam. A análise da utilização de dispositivos de proteção solar externa evidencia, também, uma semelhança entre as taxas médias de aumento de edifícios protegidos e desprotegidos. Em 2020 e em 2050 os edifícios desprotegidos apresentaram, em média, maiores taxas de aumento do consumo. Em 2080 essa situação é invertida e edifícios desprotegidos passam a apresentar taxas de aumento, em média, menores que dos edifícios protegidos.

Alguns edifícios apresentaram, ainda, comportamentos distintos na variação do consumo de energia ao avançar do presente para o futuro. A maior variação nas taxas de aumento, conforme se avança de 2020 para 2050, ocorreu justamente em edifícios com dispositivos de proteção solar (Figura 10). A menor variação ocorreu no edifício orientado a norte e sul, com $90 \%$ de abertura e sem proteção solar, um indício de que esse modelo apresenta maior resiliência, ou seja, maior capacidade de adaptação às condições futuras.

A evolução no consumo do período de 2050 ao período de 2080 evidencia maior variação nas taxas de aumento dos edifícios protegidos orientados a norte e sul (Figura 10). Nesse período, os edifícios desprotegidos e com $90 \%$ de abertura sofreram as menores variações nas taxas de aumento do consumo.

\section{Conclusões}

Apesar do esforço por parte de instituições brasileiras na busca pelo entendimento das mudanças climáticas e suas consequências para o território brasileiro, para interpretação das relações entre o clima futuro e o ambiente edificado é imprescindível o desenvolvimento de ferramentas adequadas às pesquisas dessa área. Sabe-se que os dados de saída gerados pelos modelos climáticos globais provêm de uma simplificação do clima em uma escala que não permite o detalhamento em níveis regionais. $\mathrm{O}$ acesso a modelos climáticos regionais que forneçam as informações necessárias à formatação de arquivos climáticos de entrada em softwares de simulação termoenergética de edificações é o primeiro entrave a ser solucionado para o desenvolvimento desse campo do conhecimento no Brasil. A disponibilização de dados de um modelo climático regional elevaria o nível das pesquisas, diminuindo o risco e as incertezas naturais em estudos sobre mudanças climáticas.

Figura 7 - Aumento médio mensal do consumo e aumento da temperatura de bulbo seco em 2020, 2050 e 2080

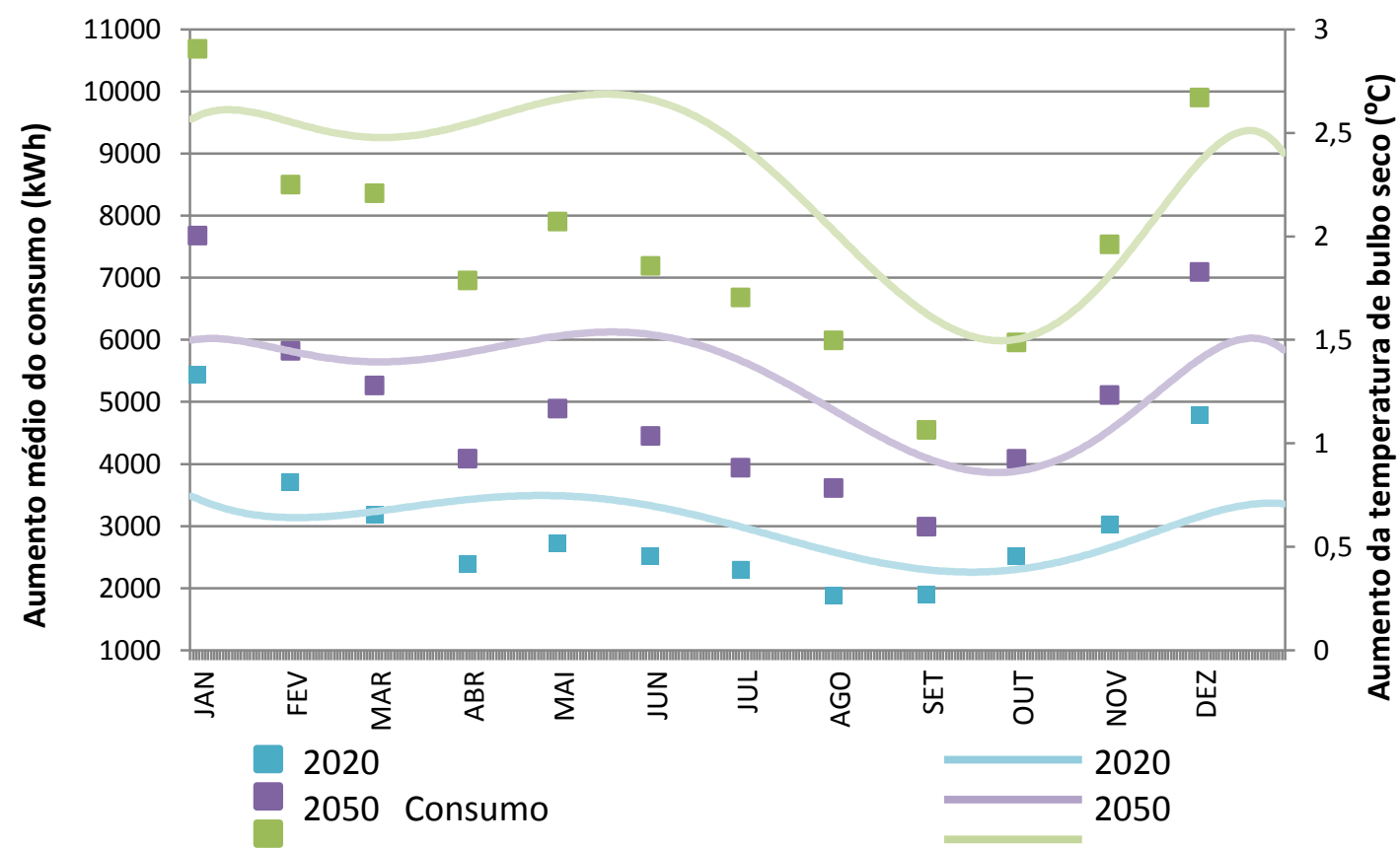


Figura 8 - Consumo de energia anual total de cada um dos modelos simulados nos quatro períodos atual, 2020, 2050 e 2080

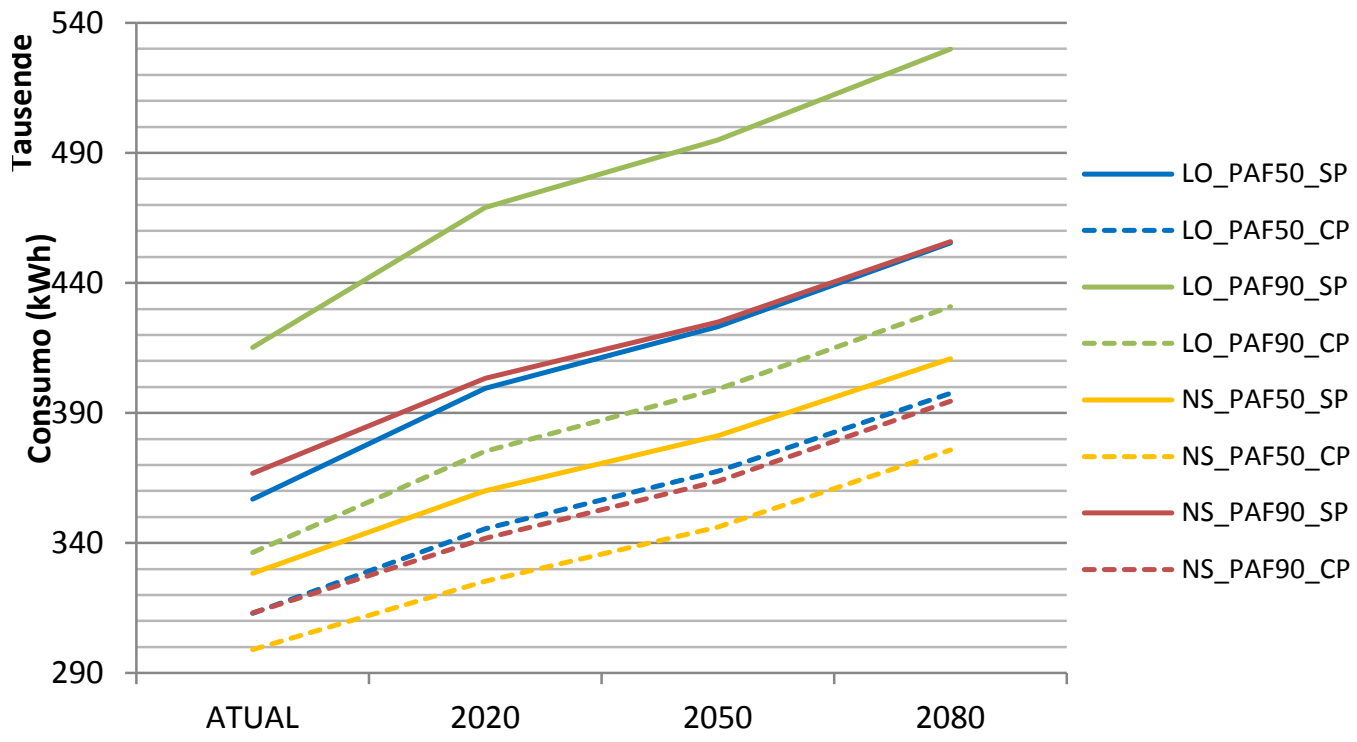

Figura 9 - Percentual de aumento no consumo de energia em 2020, 2050 e 2080, em relação ao período atual, dos modelos simulados, organizados conforme a orientação, o percentual de abertura e a proteção solar

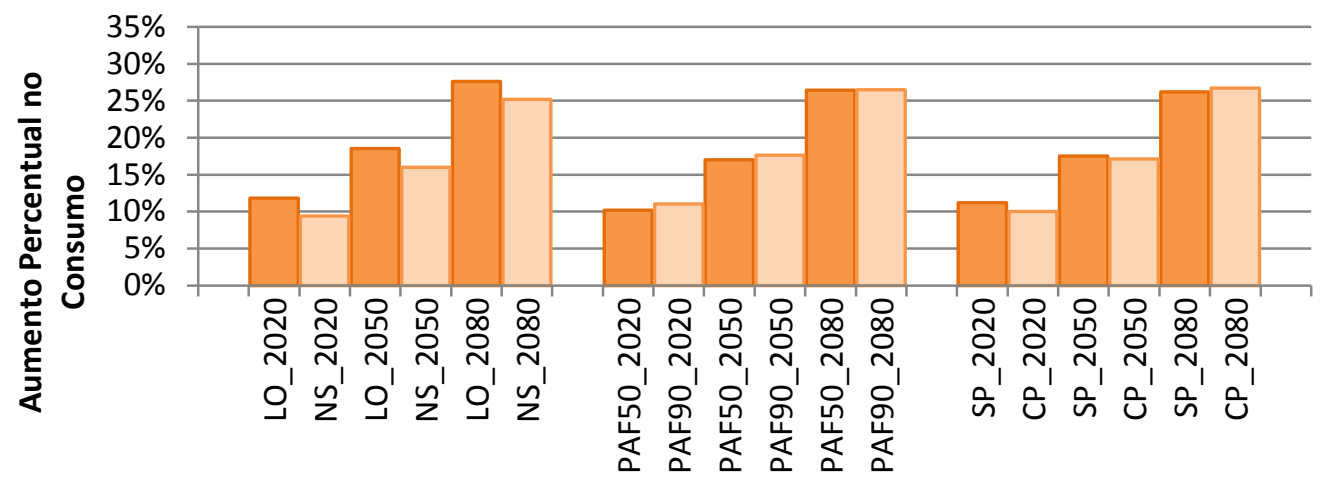

Figura 10 - Percentual de aumento no consumo de energia anual de cada um dos modelos, em relação ao período atual

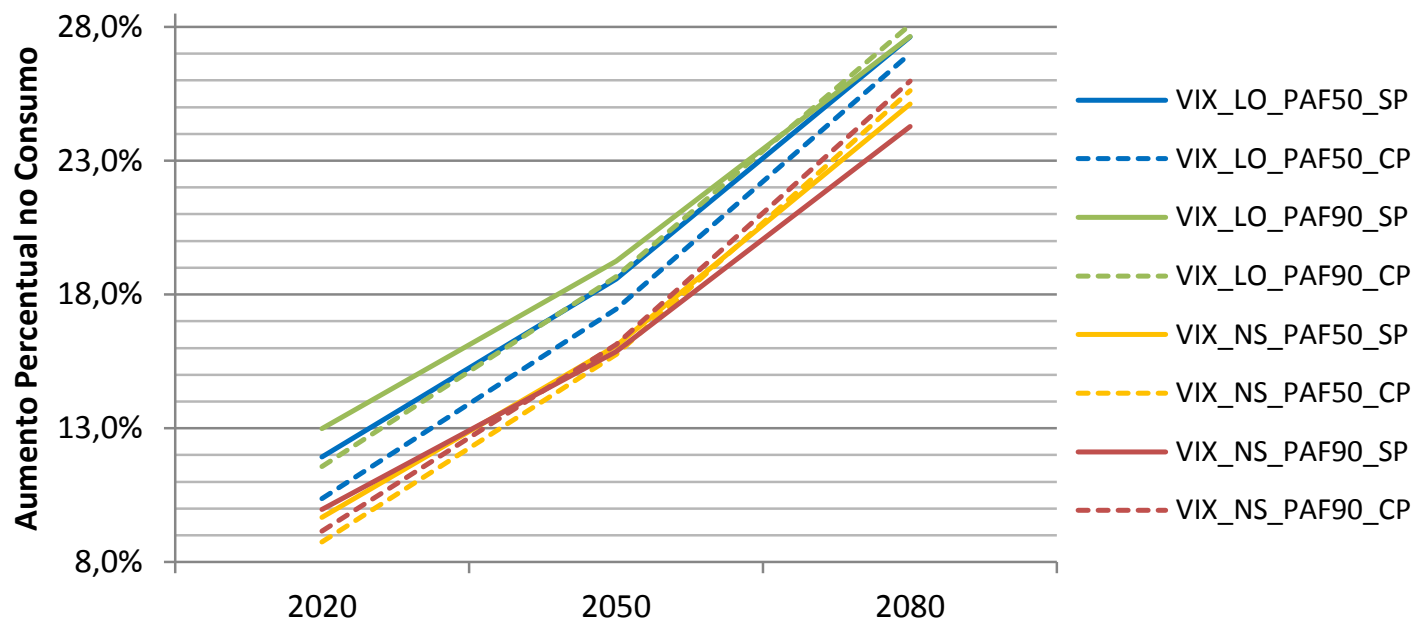

184 Casagrande, B. G.; Alvarez, C. E. de. 
Apesar dessas incertezas e da impossibilidade de teste dos resultados, diversas pesquisas ressaltam a importância da consideração das projeções climáticas futuras, não só em estudos sobre eficiência energética em edificações como também em projetos e políticas que proponham as medidas necessárias para a adaptação desse setor aos novos cenários. No caso de estudos por meio de simulações computacionais, os arquivos climáticos utilizados atualmente, referentes a períodos passados, também agregam certo nível de incertezas às pesquisas, principalmente pelo período de coleta de dados, consideravelmente distante do atual.

Nesta pesquisa foi apresentada uma revisão bibliográfica dos principais estudos sobre impactos das mudanças climáticas em edifícios, com ênfase na preparação de arquivos climáticos futuros. A partir da metodologia morphing foram formatados arquivos para três períodos futuros - 2020, 2050 e 2080 - para a cidade de Vitória, ES, aplicados em um estudo de caso de um edifício comercial.

Considerando-se o conjunto dos oito modelos, observou-se um aumento médio no consumo anual de $10,7 \%$ para o período de 2020 , de $17,3 \%$ para o período de 2050 e de $26,5 \%$ para o período de 2080, em relação ao consumo atual. Apesar de a evolução do aumento no consumo de energia apresentar um comportamento semelhante, os edifícios orientados a norte e sul mostram menores taxas de aumento que os edifícios orientados a leste e oeste.

Embora os modelos com $90 \%$ de abertura apresentem percentual de aumento no consumo relativamente maior, essa diferença é pouco representativa, principalmente em 2080. Por outro lado, os edifícios com dispositivos de proteção solar externa tendem a consumir menos energia quando comparados aos edifícios sem proteção. Porém, essa tendência se inverte em 2080, quando edifícios protegidos tem um aumento relativo no consumo maior que edifícios desprotegidos. Sobre as causas para essa ocorrência, apesar da realização de alguns testes e novas simulações, os resultados ainda não permitem afirmações conclusivas, sendo necessários aprofundamentos nesses estudos.

Alguns edifícios apresentaram, ainda, comportamentos distintos na variação do consumo de energia ao avançar do presente para o futuro; a maior variação nas taxas de aumento ocorreu justamente em edifícios protegidos. A evolução no consumo do período de 2050 a 2080 evidencia uma maior variação nas taxas de aumento dos edifícios que apresentaram menor consumo inicial: orientados a norte e sul, com dispositivos de proteção solar.

\section{Referências}

AMBRIZZI, T.; ARTAXO, P. Aquecimento Global: menos mito e mais ciência. Jornal da USP, São Paulo, p. 2, 10 set. 2012.

\section{ASSOCIAÇÃO BRASILEIRA DE NORMAS}

TÉCNICAS. NBR 15220-2: desempenho térmico de edificações: parte 2: métodos de cálculo da transmitância térmica, da capacidade térmica, do atraso térmico e do fator solar de elementos e componentes de edificações. Rio de Janeiro, 2005.

BARROS, V. Energia e Gerenciamento de Água e Mudanças Globais. In: DINIZ, E. M. Um diálogo Interdisciplinar Sobre Mudanças Globais.

Disponível em:

<http://www.iea.usp.br/iea/textos/relatorio2confre gmudancasglobaisal.pdf $>$. Acesso em: 15 set. 2012.

BELCHER, S. E.; HACKER, J. N.; POWELL, D. S. Constructing Design Weather Data For Future Climates. Building Services Engineering Research and Technology, v. 26, n. 1, p. 49-61, 2005.

BERNABÉ, A. C. A. A influência da Envoltória no Consumo Energético em Edifícios Comerciais Artificialmente Climatizados na Cidade de Vitória-ES. Vitória, 2012. Dissertação (Mestrado em Engenharia Civil) - Programa de Pós-Graduação em Engenharia Civil, Universidade Federal do Espírito Santo, Vitória, 2012.

CENTRO DE PREVISÃO DO TEMPO E ESTUDOS CLIMÁTICOS. Boletim do Projeto Uso de Cenários de Mudanças Climáticas Regionais em Estudos de Vulnerabilidade e Adaptação no Brasil e na América do Sul. Rio de Janeiro: INPE, 2005. v. 1.

CHAN, A. L. S. Developing a Modified Typical Meteorological Year Weather File For Hong Kong Taking Into Account the Urban Heat Island Effect. Building and Environment, v. 46, p. 2434-2441, 2011a.

CHAN, A. L. S. Developing Future Hourly Weather Files For Studying the Impact of Climate Change on Building Energy Performance in Hong Kong. Energy and Buildings, v. 43, p. 28602868, 2011b. 
CONFALONIERI, U. E. C. Mudança Climática Regional e Saúde. In: DINIZ, E. M. Um Diálogo Interdisciplinar Sobre Mudanças Globais. Disponível em: <http://www.iea.usp.br/iea/textos/relatorio2confre gmudancasglobaisal.pdf $>$. Acesso em: 15 set. 2012.

CRAWLEY, D. B. Creating Weather Files For Climate Change and Urbanization Impacts Analysis. Building Simulation, p. 1075-1082, 2007.

DE WILDE, P.; TIAN, W. Preliminary Application of a Methodology For Risk Assessment of Thermal Failures in Buildings Subject to Climate Change. Building Simulation, p. 2077-2084, 2009.

DE WILDE, P.; TIAN, W. Towards Probabilistic Performance Metrics For Climate Change Impact Studies. Energy and Buildings, v. 43, n. 11, p. 3013-3018, 2011.

DU, H.; EDGE, J.; UNDERWOOD, C. Modelling the Impacts of New UK Future Weather Data on a School Building. Building Simulation, p. 538545, 2011.

EAMES, M.; KERSHAW, T.; COLEY, D. A Comparison of Future Weather Created From Morphed Observed Weather and Created by a Weather Generator. Building and Environment, v. 56, p. 252-264, oct. 2012.

FREITAS, E. D. de; AMBRIZZI, T. Impacto da Rio-92 na Produção Científica da USP Considerando o Tópico Mudanças Climáticas. Estudos Avançados, v. 26, p. 341-349, 2012.

GATERELL; M. R.; MCEVOY, M. E. The Impact of Climate Change Uncertainties on the Performance of Energy Efficiency Measures Applied to Dwellings. Energy and Buildings, v. 37, n. 9, p. 982-995, 2005.

GUAN, L. Preparation of Future Weather Data to Study the Impact of Climate Change on Buildings. Building and Environment, v. 44, n. 4, p. 793800, 2009.

HANBY, V. I.; SMITH, S. T. H. Simulation of the Future Performance of Low-Energy Evaporative Cooling Systems Using UKCP09 Climate Projections. Building and Environment, v. 55, p. 110-116, 2012.

\section{INSTITUTO NACIONAL DE CIÊNCIA E} TECNOLOGIA. Instituto Nacional de Ciência e Tecnologia da Criosfera. Disponível em: <http://memoria.cnpq.br/programas/inct/_apresent acao/inct_criosfera.html>. Acesso em: 25 jan. 2013.
INTERGOVERNMENTAL PANEL ON CLIMATE CHANGE. Understanding climate change: 22 years of IPCC assessment. IPCC, 2010.

JENTSCH, M. F.; BAHAJ, A. S.; JAMES, P. A. B. Climate Change Future Proofing of Buildings: generation and assessment of building simulation weather files. Energy and Buildings, v. 40, n. 12, p. 2148-2168, 2008.

KERSHAW, T.; EAMES, M.; COLEY, D. Assessing the Risk of Climate Change For Buildings: a comparison between multi-year and probabilistic reference year simulations. Building and Environment, v. 46, n. 6, p. 1303-1308, 2011.

KOLOKOTRONI, M. et al. London"s Urban Heat Iisland: impact on current and future energy consumption in office buildings. Energy and Buildings, v. 47, p. 302-311, 2012.

LABORATÓRIO DE EFICIÊNCIA ENERGÉTICA EM EDIFICAÇÕES. Relatório 200504: processamento de arquivos climáticos para simulação do desempenho energético de edificações. Florianópolis: 2005. Disponível em: <http://www.labeee.ufsc.br/sites/default/files/arqui vos_climaticos/RT200504.pdf>. Acesso em: 05 maio 2012.

LAMBERTS, R.; GHISI, E.; RAMOS, G. (Orgs.). Impactos da Adequação Climática Sobre a Eficiência Energética e o Conforto Térmico de Edifícios de Escritórios no Brasil. Florianópolis: LABEEE, 2006.

LEE, T. Changing Climate: ersatz future weather data for lifelong system evaluation. Building Simulation, p. 633-640, 2011.

MARENGO, J. A. Mudanças Climáticas Globais e Efeitos Sobre a Biodiversidade: caracterização do clima atual e definição das alterações climáticas para o território brasileiro ao longo do Século XXI. Brasília: MMA, 2007.

MINISTÉRIO DE MINAS E ENERGIA. EMPRESA DE PESQUISA ENERGÉTICA. Balanço Energético Nacional 2011: ano base 2010. Rio de Janeiro: EPE, 2011.

MINISTÉRIO DE MINAS E ENERGIA. Etiquetagem de Eficiência Energética em Edificações. Rio de Janeiro: Procel/Eletrobras, 2009a.

MINISTÉRIO DE MINAS E ENERGIA. Manual Para Aplicação dos Regulamentos: RTQ-C e RAC-C. Rio de Janeiro: Procel/Eletrobras, 2009b. 
OUEDRAOGO, B. I.; LEVERMORE, G. J.;

PARKINSON, J. B. Future Energy Demand For Public Buildings in the Context of Climate Change for Burkina Faso. Building and Environment, v. 49, p. 270-282, 2012.

PITA, M. F. Los Cambios Climáticos. In: CUADRAT, J. M.; PITA, M. F. Climatología. 4. ed. Madrid: Cátedra, 2006.

ROBERT, A.; KUMMERT, M. Designing NetZero Energy Buildings For the Future Cclimate, Not For the Past. Building and Environment, v. 55, p. 150-158, sep. 2012.

UNITED STATES DEPARTMENT OF ENERGY. Energy Efficiency and Renewable Energy. EnergyPlus Energy Simulation Software. Disponível em: <http://apps1.eere.energy.gov/buildings/tools_dire ctory/>. Acesso em: 15 mar. 2013.
WANG, X.; CHEN, D.; REN, Z. Assessment of climate change impact on residential building heating and cooling energy requirement in Australia. Building and Environment, n. 45, p. 1663-1682, 2010.

WANG, X.; CHEN, D.; REN, Z. Global warming and its implication to emission reduction strategies for residential buildings. Building and Environment, n. 46, p. 871-883, 2011.

\section{Agradecimentos}

Ao Conselho Nacional de Desenvolvimento Científico e Tecnológico (CNPq), pelo apoio financeiro para a realização desta pesquisa.

Bruna Gomes Casagrande

Programa de Pós-Graduação em Engenharia Civil, Centro Tecnológico | Universidade Federal do Espírito Santo | AV. Fernando Ferrari, 514, Prédio CT VI, sala 204, Goiabeiras | Vitória - ES - Brasil | CEP 29075-910 | Tel.: (27) 4009-2652 |

E-mail: casagrandebruna@yahoo.com.br

\section{Cristina Engel de Alvarez}

Laboratório de Planejamento e Projetos, Centro de Artes | Universidade Federal do Espírito Santo | Tel.: (27) 4009-2581 |

E-mail: cristina.engel@ufes.br

\section{Revista Ambiente Construído}

Associação Nacional de Tecnologia do Ambiente Construído

Av. Osvaldo Aranha, $99-3^{\circ}$ andar, Centro

Porto Alegre - RS - Brasil

CEP 90035-190

Telefone: +55 (51) 3308-4084

Fax: +55 (51) 3308-4054

www.seer.ufrgs.br/ambienteconstruido

E-mail: ambienteconstruido@ufrgs.br 